\title{
Alu Element
}

National Cancer Institute

\section{Source}

National Cancer Institute. Alu Element. NCI Thesaurus. Code C129404.

A family of short interspersed nuclear elements found in the human genome that are substrates for the Arthrobacter luteus restriction endonuclease. Alu elements are highly conserved within primate genomes and have been used in the investigation of human population genetics, primate and human evolution, and human diseases, including cancer. 